\title{
Partially (in)visible Higgs decays at the LHC
}

\author{
Christoph Englert, ${ }^{1}$ Michael Spannowsky, ${ }^{1}$ and Chris Wymant ${ }^{1}$ \\ ${ }^{1}$ Institute for Particle Physics Phenomenology, Department of Physics, \\ Durham University, DH1 3LE, United Kingdom
}

\begin{abstract}
Both Atlas and CMS have reported a discovery of a Standard Model-like Higgs boson $H$ of mass around $125 \mathrm{GeV}$. Consistency with the Standard Model implies the non-observation of non-SM like decay modes of the newly discovered particle. Sensitivity to such decay modes, especially when they involve partially invisible final states is currently beyond scrutiny of the LHC. We systematically study such decay channels in the form of $H \rightarrow A A \rightarrow$ jets + missing energy, with $A$ a light scalar or scalar, and analyze to what extent these exotic branching fractions can be constrained by direct measurements at the LHC. While the analysis is challenging, constraints as good as BR $\lesssim 10 \%$ can be obtained.
\end{abstract}

\section{INTRODUCTION}

Recent results obtained at the Large Hadron Collider by the Atlas and CMS experiments [1, 2] have revealed the existence of a light Higgs candidate [3] with a mass of $\sim 125 \mathrm{GeV}$. The observation of this new particle combines evidence in the $H \rightarrow \gamma \gamma, H \rightarrow Z Z$ and $H \rightarrow W^{+} W^{-}$channels. Given the yet small collected luminosity, the properties of this newly discovered resonance are still subject to large statistical uncertainties [4]. Analyses targeting e.g. spin and $\mathcal{C P}[5,6]$ of the new particle and a more precise extraction of its couplings to known matter will be addressed with a larger data sample.

The current observations leave open a plurality of phenomenological possibilities of Higgs sectormodifications [7, 8]. Especially the extraction of the resonance's couplings is influenced by non-standard decays [9-13] since it is based on a fit to combinations of various production $p$ and decay modes $d$. These are functions of the partial and total decay widths and all couplings $\left\{g_{i}\right\}$ :

$$
\sigma_{p} \times \mathrm{BR}_{d} \sim \frac{\Gamma_{p} \Gamma_{d}}{\Gamma_{\text {tot }}} \sim g_{p}^{2} g_{d}^{2} /\left(\sum_{\text {modes }} g_{i}^{2}\right) .
$$

The total width $\Gamma_{\text {tot }}$ in current fits is typically approximated by including a freely flowing invisible partial width [14] to the list of decay modes or by imposing the constraint $\Gamma_{\text {invis }} \sim$ $g_{\text {invis }}^{2}=0$.

Extracting such an invisible* or partially visible branching ratio is an experimentally ambitious task. The decay of the Higgs via a light scalar or pseudo-scalar $A$ can be buried in a large hadronic background, experimental systematics can limit the sensitivity to such decays, and non-SM phenomenology can easily be missed. The signature $H \rightarrow A A$ occurs in many extensions of the SM, e.g., the next-to-minimal supersymmetric Standard Model [15], Higgs-portal models [16], and whenever an approximate symmetry of the Higgs potential is explicitly broken by a small term in the potential, giving a light pseudo-Nambu-Goldstone boson (see [17] for a clear

\footnotetext{
*Note that from Eq. 11 "invisible" also means fully visible in a non-standard search channel.
}

discussion). However the signature can be missed by standard searches, depending on how $A$ itself decays, and new dedicated strategies need to be devised. Obviously, an observation of such a novel decay channel $H \rightarrow A A$ would directly imply physics beyond the Standard Model.

Subjet methods (pioneered in Ref. [18]) have proven particularly successful in getting a handle on such a modified phenomenology. In particular, subjet analyses applied to the decay chains $H \rightarrow 2 A \rightarrow 4 X$ have unravelled potential sensitivity to these non-standard decays if the (pseudo)scalar $A$ is light $\mathcal{O}(10-20 \mathrm{GeV})$. A decay of the Higgs to $A A$ is well motivated on general grounds; one can keep a reasonably open mind with regards to how the $A A$ subsequently decay. $H \rightarrow A A \rightarrow 4 g$ is considered in [19], $H \rightarrow A A \rightarrow 4 \tau$ in [20], $H \rightarrow 2 A \rightarrow 2 \tau 2 \mu$ in [17] and $H \rightarrow 2 A \rightarrow 4 c$ in [21]. Higgs decays into resonances with masses close to hadronic bound states have been studied in Refs. [10, 22]. Sensitivity to the signatures discussed in Refs. [10, 17, 19-22] follows from fundamentally distinct QCD and electroweak properties, highlighting the diverse power that jet substructure-based approaches offer.

There is one major difference in the analysis of purely hadronic final states compared to electroweak final states. Quite often the latter involve a significant amount of missing energy, which is aligned with the direction of the fat jet that is input to grooming and/or tagging algorithms [23]. Normally such an event topology is avoided to minimize systematic uncertainties. In, e.g., searches for supersymmetry in the jets+missing energy channel [24] one requires a missing energy vector $\mathbb{E}_{T}$ well isolated from a number of hard jets, to reduce systematics. Decays $H \rightarrow A A$ with $m_{H} / m_{A} \gg 2$, on the other hand, naturally involve non-isolation of $\mathbb{E}_{T}$, which might even be not too large depending on the decay of $A$. In the phase space region where we can separate signal from background a SUSY-inspired search strategy based on a strong $\mathbb{E}_{T}$ isolation becomes impossible.

Strategies to deal with non-SM Higgs decays involving both missing and hadronic energy [25] are, hence, limited. A direct generalization of the standard invisible Higgs decay search in the weak boson fusion channel [11] is not possible. Such an analysis relies on a central jet veto to obtain a sufficiently large signal-over-background ratio $S / B$ to study the jets' azimuthal angle correlation. Applying a central jet veto 

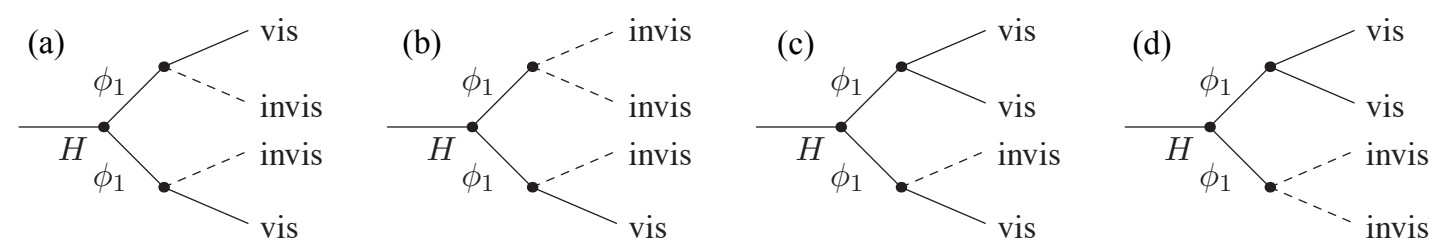

FIG. 1: Higgs decay topologies in the simplified models that we study for the purpose of this paper. "vis" is a placeholder for $u \bar{u}, d \bar{d}$ flavor quark pairs that give rise to visible hadronic energy.

to central decay $H \rightarrow \mathbb{E}_{T}$ +hadrons removes the signal that we would like to investigate.

Adapted Higgs+monojet searches [9, 13] are challenged by overwhelmingly large dijet and weak boson+jet backgrounds and trigger issues, as soon as missing energy from invisible Higgs decays decreases when turning to a partially visible decay. The latter can at least be partially cured by focussing on the Higgs' $p_{T}$-distribution's tail. This, however, comes at the price of larger theoretical and experimental uncertainties, which imperatively need to be included to reach a realistic formulation of the branching ratio constraints.

This leaves Higgs-strahlung $p p \rightarrow H Z$ as the bestmotivated channel to study the situation we have in mind at the LHC. Trigger issues are avoided by reconstructing the leptonic $Z \rightarrow e^{+} e^{-}, \mu^{+} \mu^{-}$decay and no further adjustments to the trigger settings or thresholds are necessary to perform the measurement(this process is also studied in the context of invisible Higgs searches [26]). Furthermore, the jet energy scale calibration is performed with $Z+$ jet events, whose distribution is both theoretically and experimentally under good control [27, 28]. Data-driven methods can be straightforwardly applied in "ABCD" approaches, when e.g. comparing boosted to the un-boosted $Z$ boson kinematics.

\section{DECAY TOPOLOGIES}

We employ a simplified-model based approach [29] to investigate the LHC search potential to the $H \rightarrow \mathbb{E}_{T}+$ hadrons signature. More precisely we study the Higgs decay realizations depicted in Fig. 11 These scenarios (a)-(d) are characterized by different kinematics and different relative contributions of missing and hadronic energy in the final state.

We limit our analysis to the light flavor final states $u, d$; particles with $\sim 10 \mathrm{GeV}$ masses and a significant coupling to $b$ quarks are not only constrained by upsilon measurements [30], but also give rise to plethora of dedicated phenomenological handles on the final state [22, 31, 32] that we do not wish to exploit to be as general as possible. Similar arguments hold for the $H \rightarrow 4 \tau$ decay, which gives rise to sparse but focused hadronic energy deposits [20]. Both these cases (and also $H \rightarrow 4 g$ ) are fundamentally different from the topologies of Fig. 1 where "visible" refers to light flavor quarks. The quarks undergo normal showering and hadronization leaving neither the possibility for flavor tags nor for detecting pronged decays from counting charged tracks, as done in the $b$ - and $\tau$-flavored decays of $H \rightarrow A A$, respectively.

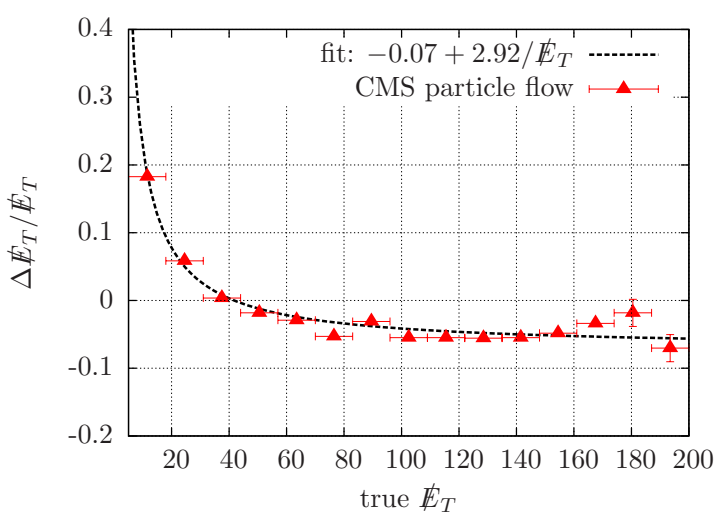

FIG. 2: $\Delta \mathbb{E}_{T} / \mathbb{E}_{T}$ as a function of the true $\mathbb{E}_{T}$ that follows from particle flow and early LHC data [39]. We also display the fitted function that is employed for our analysis.

\section{ELEMENTS OF THE ANALYSIS}

We implement the decay topologies using FEYNRULES [33] and use its interface to SHERPA [34, 35] to generate events for the mass choices $m_{H}=125 \mathrm{GeV}, m_{A}=$ $20 \mathrm{GeV}, m_{\text {invis }}=10 \mathrm{GeV}$. This choice is not special and the details of eventually extracting the branching ratio is not sensitive to the particular value $m_{A}$ unless $m_{H} \ngtr m_{A}$. We will comment on the possibility to extract $m_{A}$ in Sec.IV.

We generate background events using SHERPA and include $W W+$ jet, $W Z+$ jet, $Z Z+$ jet, and $t \bar{t}+$ jets as the main backgrounds to our $p p \rightarrow\left(Z \rightarrow \ell^{+} \ell^{-}\right)+$jet $+\mathbb{E}_{T}$ analysis. We normalize our signal and background event samples to the corresponding higher order-corrected cross sections [36-38]. Studying the impact of a mismeasurement of $Z+$ jets events requires the simulation of a realistic detector environment, and should be addressed by the experiments. However, we may assume on the basis of Refs. [27, 28] that this background can be brought under sufficient control and can be subtracted from the eventual distribution also when the missing energy vector is collinear to the jet. We include a flat shape uncertainty of the background distribution to partly account for the jet energy scale uncertainty in the computation of the expected BR limits in Sec.IV

Associated Higgs production with SM-like Higgs decays to $b \bar{b}$ and $\tau \tau$ also comprise backgrounds to our $\left(Z \rightarrow \ell^{+} \ell^{-}\right)+$ $\mathbb{E}_{T}+$ jet analysis and we include them consistently throughout. 


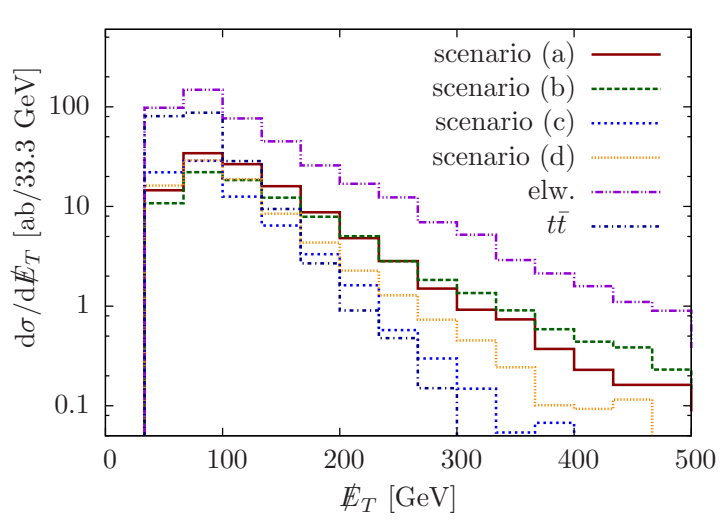

(a)

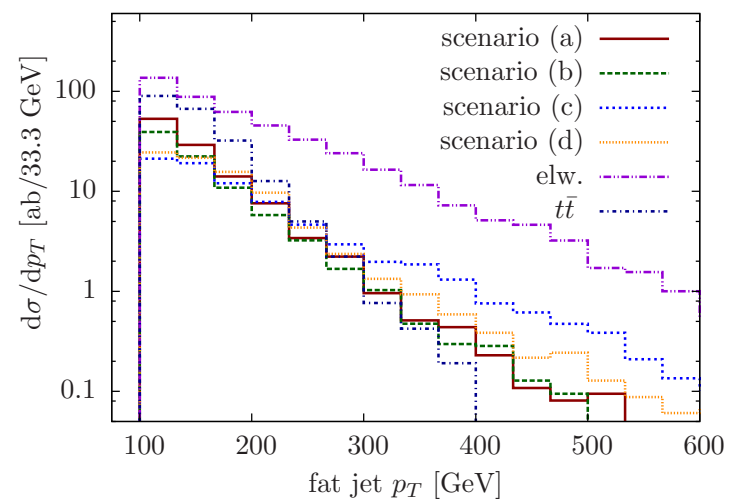

(b)

FIG. 3: Missing transverse energy (a) and fat jet transverse momentum (b) of the decay scenarios Fig.11a)-(d) and the contributing backgrounds after all analysis steps have been applied.

We reconstruct the events' visible final states using a hybrid ECAL + HCAL implementation which granularizes the final state particles on grids with $\Delta \eta \times \Delta \phi=0.025 \times 0.025(0.1 \times$ 0.1) ECAL (HCAL) as massless cell entries and feed the reconstructed objects to a smearing routine which mimics detector effects as described later on. Doing so, we reconstruct the full three-momenta from the detector geometry with an invariant mass $p^{2}=0$ for each ECAL+HCAL cell, which contains a single or multiple hits.

In the analysis, we first reconstruct isolated stable leptons, by requiring the hadronic energy deposit in the vicinity of the lepton candidate $\left(\Delta R=\sqrt{\Delta \eta^{2}+\Delta \phi^{2}} \leq 0.1\right.$, where $\eta$, and $\phi$ are pseudorapidity and azimuthal angle respectively) to be smaller than $10 \%$ of the lepton candidate's transverse momentum. We furthermore require exactly two leptons $\left(p_{T, \ell}>10 \mathrm{GeV},\left|\eta_{\ell}\right|<2.5\right)$ of identical flavor and opposite charge that recombine the $Z$ mass within $m_{Z} \pm 10 \mathrm{GeV}$ (note that this also removes higher-pronged $H \rightarrow \tau \tau$ events) and demand $p_{T, Z}=\left(p_{\ell_{1}}+p_{\ell_{2}}\right)_{T}>130 \mathrm{GeV}$. Subsequently, we cluster Cambridge/Aachen jets with $R=1.5$ using FASTJET [40] and we require at least one such fat jet with $p_{T}>100 \mathrm{GeV}$. We apply the mass drop and asymmetry criteria to this jet as explained in Ref. [18], we filter and trim it [41], and we keep the three hardest subjets [18]. Furthermore, the jet needs to pass a $b$-veto in $|\eta|<2.5$, for which we choose a working point [42] with a flat $70 \%$ tagging efficiency and a flat $10 \%$ fake rate. The $b$ veto removes the pollution from $H, Z \rightarrow b \bar{b}$ and significantly reduces the $t \bar{t}$ background.

In the next step we remove the fat jet from the event and re-cluster the remaining tracks to anti- $k_{T}$ jets with $R=0.4$ and $p_{T}>30 \mathrm{GeV}$. In order to project out the signal region we are interested in, we require a large missing transverse energy $E_{T}>50 \mathrm{GeV}$, which is reconstructed from all visible hits. It needs to be isolated from the reconstructed $Z$ boson $R\left(\mathbb{E}_{T}, Z\right) \geq 2$, and we veto additional jets around the $Z$ : $\forall j: R(Z, j)>1.5$ to further reduce $t \bar{t}$ events.

Throughout the analysis we include detector smearing which we choose for jets and leptons according to Ref. [43]:

$$
\begin{aligned}
\text { jets : } \frac{\Delta E}{E} & =\frac{5.2}{E} \oplus \frac{0.16}{\sqrt{E}} \oplus 0.033, \\
\text { leptons : } \frac{\Delta E}{E} & =0.02
\end{aligned}
$$

and we include the missing energy response from recent particle flow fits of CMS [39] (obtained from the $7 \mathrm{TeV}$ data set, Fig. 2):

$$
\text { missing energy : } \frac{\Delta \mathbb{E}_{T}}{\mathbb{E}_{T}}=\frac{2.92}{\mathbb{E}_{T}}-0.07 .
$$

In fact, the quoted jet and lepton energy resolutions are better than the ones obtained from particle flow observables [39]. The resolution of $\mathbb{E}_{T}$ supersedes the original estimates of Ref. [43] and we can therefore assume that Eq. (2) is a conservative baseline for the $14 \mathrm{TeV}$ run at luminosities at which the limits on the BRs will be stringent. We note that the $\mathbb{E}_{T}$ resolution is the main limiting factor of the analysis that we pursue.

After applying all cuts, we are left with only minor possibilities to enhance signal over background (apart from including an invariant mass cut around the reconstructed Higgs mass, of course). Fig. 3 displays the $\mathbb{E}_{T}$ and fat jet $p_{T}$ distribution for the signal (assuming BR $=1$ to the discussed topologies) and the discussed backgrounds. With all described cuts applied, $t \bar{t}$ is sufficiently reduced, while the electroweak $V V+$ jet backgrounds are still large. Additionally, the $\mathbb{E}_{T}$ and $p_{T}$ distributions do not show any particular discriminative features, which would allow to gain in $S / B$ from tighter and harder missing energy and jet $p_{T}$ cuts (note that this especially holds when we need to relax BR $=1$ for deriving upper limits on the branching ratio). We find that SM-like Higgs decays are sufficiently suppressed to make negligible contribution to the background.

In order to derive expected limits on the branching ratios we need to reconstruct the $125 \mathrm{GeV}$ resonance in this search channel in the most efficient way. There are, in fact, dedicated 

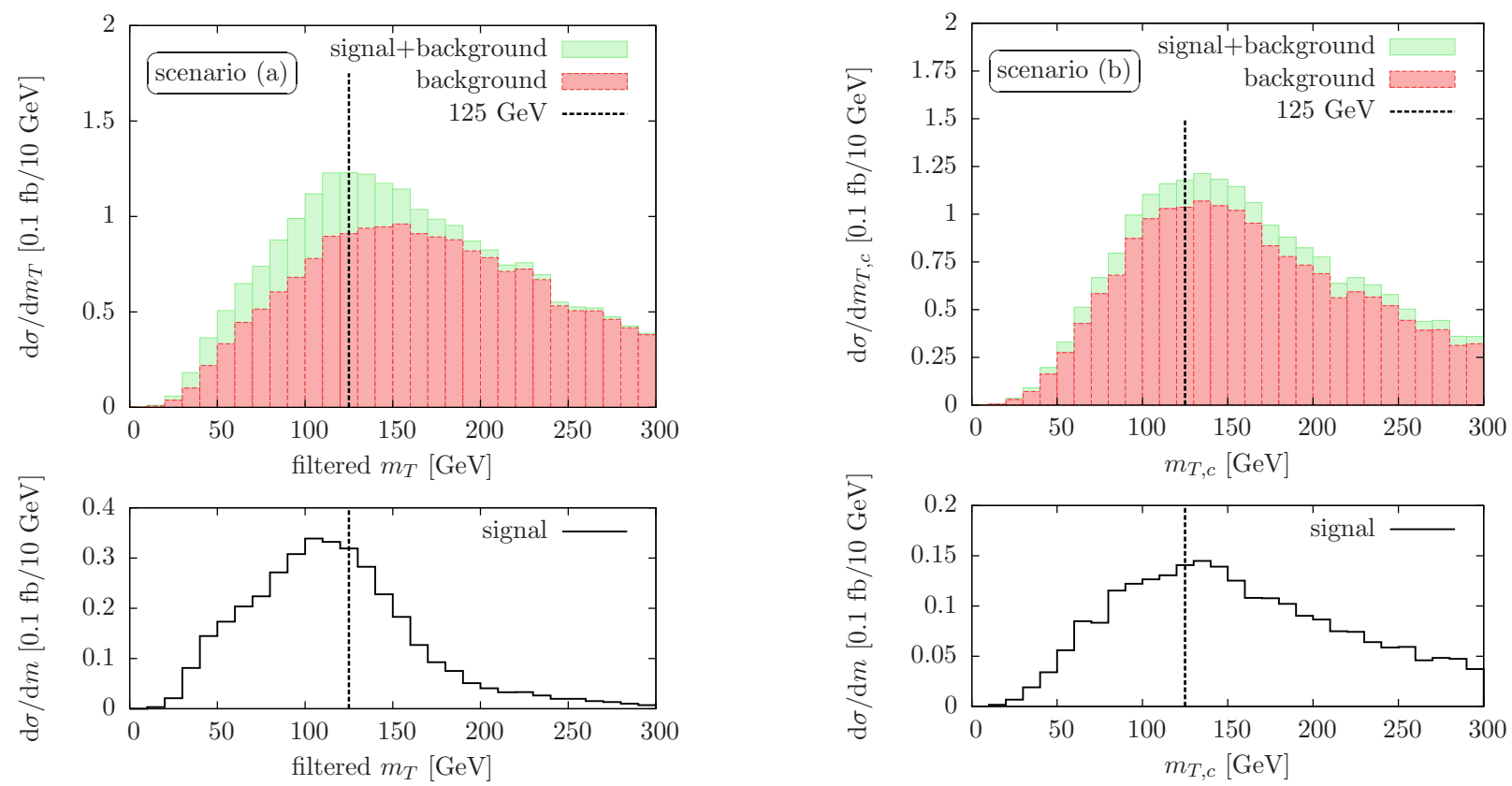

(a)
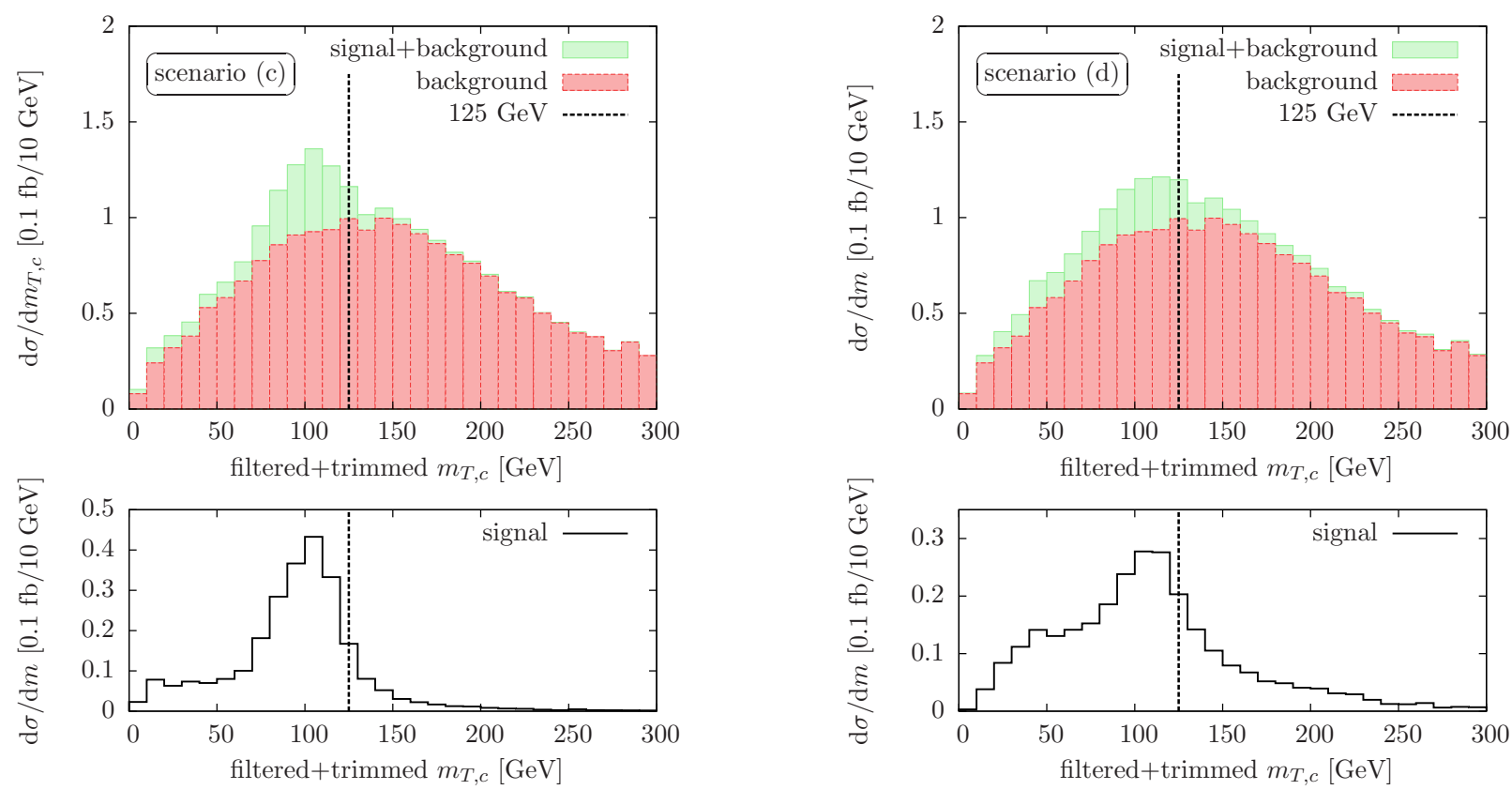

(c)

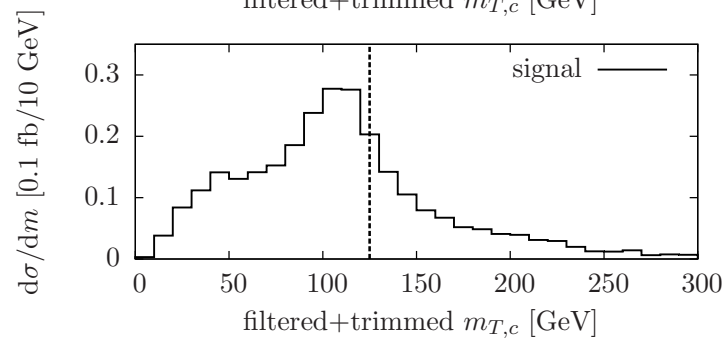

(d)

FIG. 4: Transverse mass or transverse cluster mass distributions after filtering and filtering+trimming, depending on the scenario of Fig. 11 We assume $\mathrm{BR}($ scenario $(\mathrm{i}))=1, i=(a)-(d)$ for illustration purposes.

observables, which facilitate the reconstruction of invariant mass peaks when missing energy is involved in the decay of a heavy resonance and they also prove helpful in the present context. One example which is useful for our purposes is the transverse mass definition

$$
m_{T}^{2}=\left(\sqrt{\notin_{T}^{2}+m_{j}^{2}}+\sqrt{p_{T, j}^{2}+m_{j}^{2}}\right)^{2}-\left(\mathbf{p}_{T, j}+\mathbf{p}_{T}\right)^{2}
$$

which generalizes the invariant mass definition of Ref. [44] to the present situation. In Ref. [44] an analogue definition of the invariant mass is applied to $H \rightarrow \tau \tau$ (see also [32]) with full leptonic $\tau$ decays in the collinear approximation, i.e. the individual $\not p$-lepton correlation from each $\tau$ decay is incorporated. Applied to our signatures this corresponds to a leptonic version of Fig. 11 a), and we can expect a good reconstruction of the Higgs resonance for this decay scenario. 


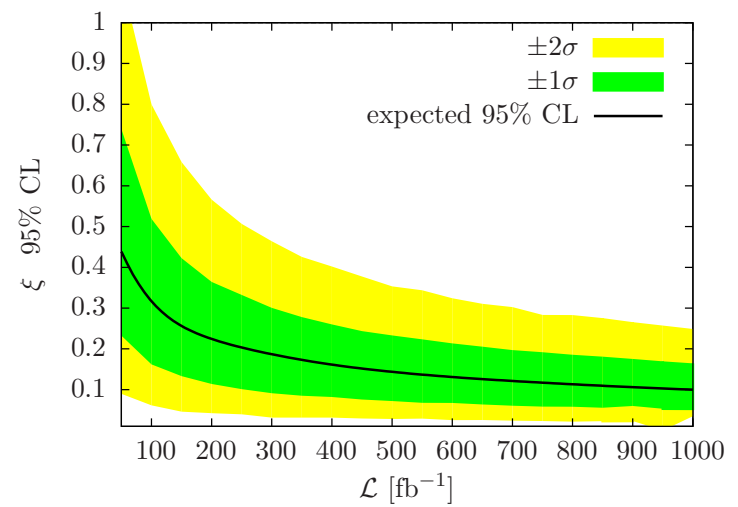

(a)

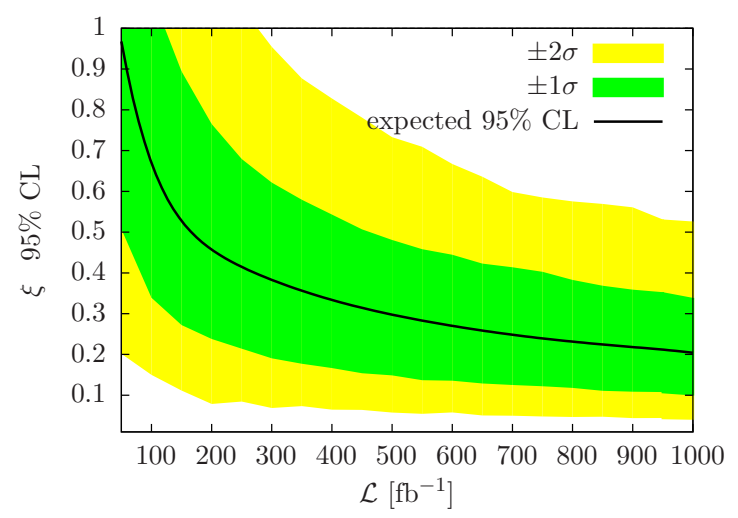

(c)

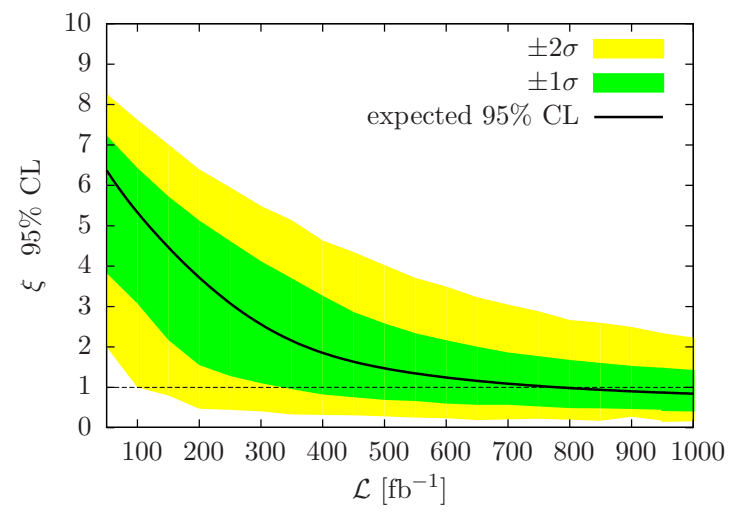

(b)

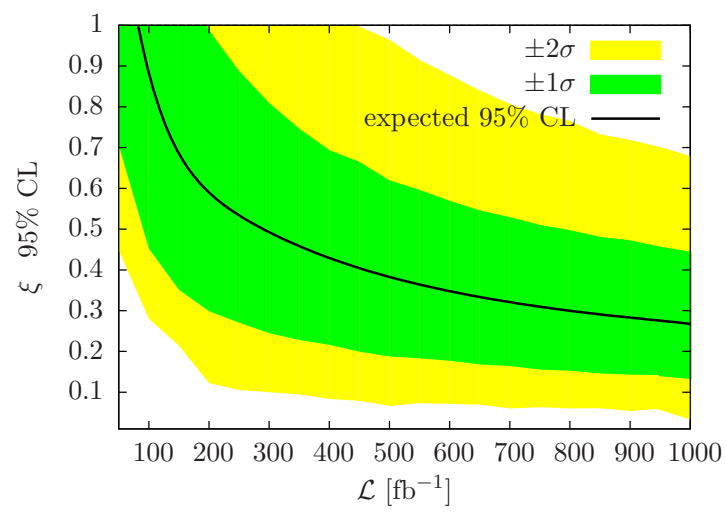

(d)

FIG. 5: 95\% confidence level exclusion of the various scenario's branching ratio (or signal strength $\xi=\sigma \times \mathrm{BR} / \sigma_{\mathrm{SM}}$ to be more specific) from a direct measurement along the lines of Sec. III We assume a background template uncertainty of initially $10 \%$, which is we assume to saturated at 5\% at end-of-lifetime LHC luminosities of $\mathcal{L}=1000 / \mathrm{fb}$.

Another observable which reconstructs the mass of a heavy decaying resonance from a kinematic endpoint measurement is the so-called transverse cluster mass [45]

$$
m_{T, c}^{2}=\left(\sqrt{m_{j}^{2}+p_{T, j}^{2}}+\mathbb{E}_{T}\right)^{2}-\left(\mathbf{p}_{T, j}+\boldsymbol{p}_{T}\right)^{2} .
$$

This observable is a good choice if there is a relatively large amount of visible energy compared to invisible energy [20, 46], as encountered in our scenarios Fig. 1]c) and (d), for which it allows to reconstruct the Higgs mass from a Jacobian peak.

For signatures involving a lot of missing energy such as Fig. (1) $(\mathrm{c})$, we need to break the degeneracy of the $\mathbb{E}_{T}$ vector to facilitate an approximate mass reconstruction. For scenario Fig. 1(b) we project $\not p$ onto the hardest reconstructed subjet after filtering $\tilde{j}$, and assign an energy $\notin=E_{\tilde{j}}$. We define a second missing energy four vector from the shift with respect to the original missing energy vector $\tilde{p}=\not p_{\text {orig }}-\not p$. This breaks the missing energy degeneracy and assumes a collinear democratic $A$ decay in the lab frame. Resolution effects have the biggest influence on this decay scenario and the mass reconstruction will be worst among the four scenarios that we discuss in this work.
The corresponding invariant mass distributions for our scenarios Fig. 11a)-(d) after all cuts have been applied are shown in Fig. 44, where we again assume $\mathrm{BR}($ scenario (i) $)=1, i=$ $(a)-(d)$. We pick the best representation of the Higgs mass peak by studying the invariant mass distributions before and after filtering and trimming. From the different jetsubstructure that follows as a consequence of the different decay topologies, trimming can remove too much of the signal when the light final state quark pairs are relatively widely separated in scenario (a).

When focussing on scenarios with an increasingly large amount of $\mathbb{E}_{T}$ compared to hadronic activity, sensitivity decreases. This is a consequence of the applied subjet analysis, which requires by definition a significant amount of a hadronic energy. Depending on the scenario we therefore become increasingly sensitive to uncorrelated initial state radiation and underlying event splash-in which degrades the mass resolution, while filtering and trimming serve to suppress part of the pollution from underlying event. 

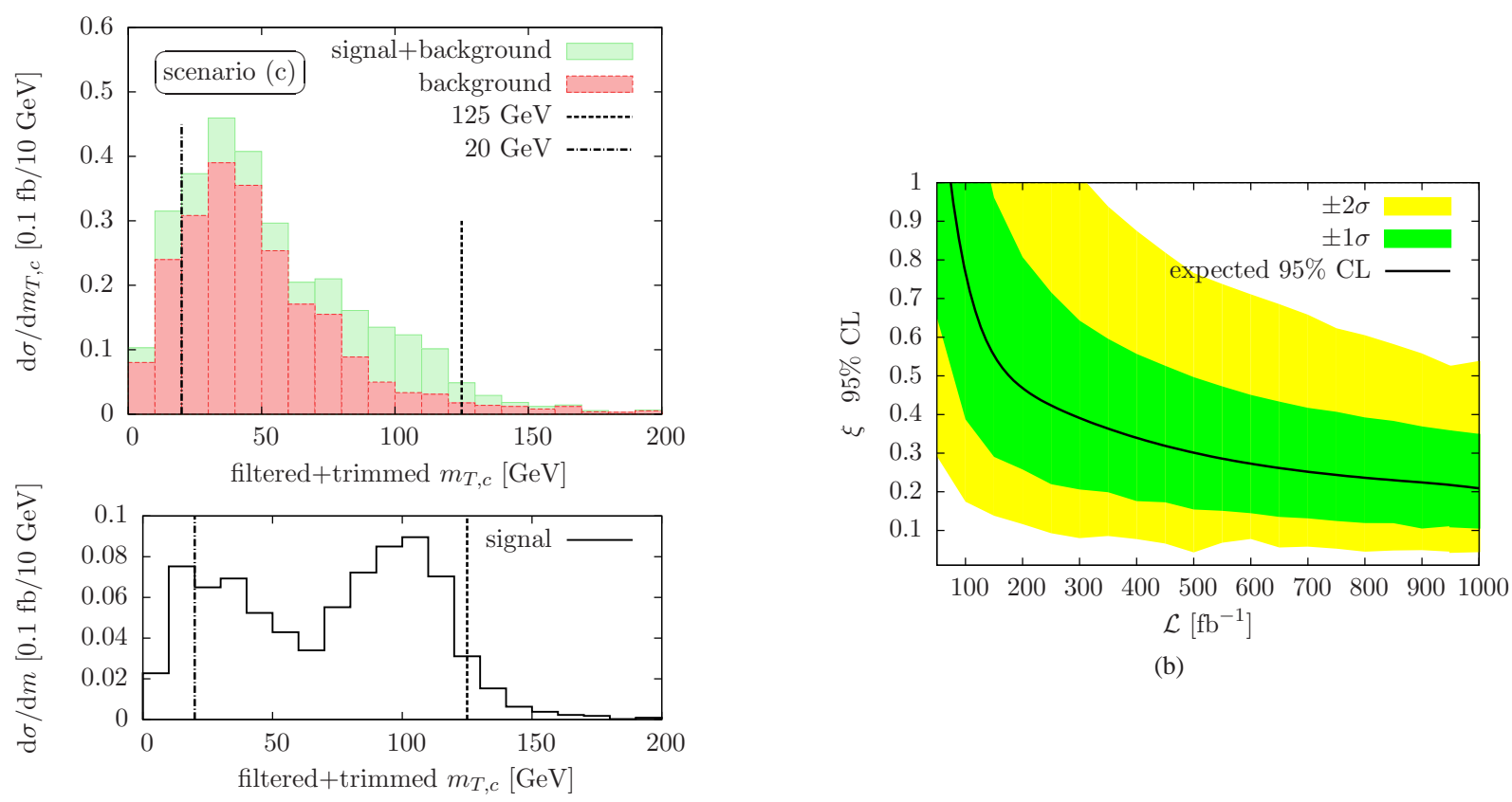

(b)

(a)

FIG. 6: (a) Transverse cluster mass for scenario (c) with the additional requirement Eq. 7), and (b) the resulting branching ratio extraction along the lines of Sec. IV

\section{BRANCHING RATIO LIMITS AT THE LHC}

We move forward and use the distributions of Fig. 4 to derive an estimate of the bounds on the branching ratios that can be obtained at the LHC $14 \mathrm{TeV}$ from direct measurements. We follow the experimentalists' approach and use the histograms of Fig. 4 as an input to the $\mathrm{CL}_{s}$ method [47, 48] to derive 95\% $\mathrm{CL}_{s}$ exclusion limits on the branching ratio using the binned log-likelihood test statistic. We include a shape uncertainty of the background template in the computation of confidence levels that eventually enter the $\mathrm{CL}_{s}$ ratio. We choose this shape uncertainty as a flat profile which we assume dependent on the integrated luminosity

$$
\Delta(\mathcal{L})=10 \%-5 \% \sqrt{\mathcal{L} / 1000 \mathrm{fb}^{-1}}
$$

to mimic an improved understanding of the measurement and theoretical uncertainty in due time.

The expected $95 \%$ confidence level exclusion is shown in Fig. 5. Direct measurements yield results $\mathrm{BR} \lesssim 0.1, \ldots, 1$, and are highly sensitive to the amount of hadronic energy that we observe in the final state and the correlation of $\mathbb{E}_{T}$ with the visible part of the final state.

We learn from Fig. 4(b) that the combination of little hadronic energy, trigger criteria and detector effects together with initial-state-radiation pollution results in an extremely challenging signature at the hadronically busy LHC environment. As a direct consequence, only weak limits can be obtained for decay scenario (b). This is mostly due to the very limited possibilities to improve $S / B$ and the poor mass res- olution that can be obtained from a signature that predominantly governed by missing energy in a channel whose diboson backgrounds' also contain a sizable amount of $\mathbb{E}_{T}$.

Turning to final states that contain more hadronic energy Fig. 1(c) and (d), we can impose stronger limits from direct measurements $(\mathrm{BR} \lesssim 0.3)$. An optimal choice of observables adopted to the specifics of Fig. 11 (a) in comparison to (c) can push the bounds to the $10 \%$ level.

An alternative route for putting limits on scenario (c), where hadronic energy correlations in the fat jet's substructure can be resolved, is exploiting the fat jet's "active area" (see also Ref. [20]) in a way that also incorporates the correlation with the missing energy. This is straightforwardly achieved by imposing an additional cut on the ratio of the fat jet's transverse momentum and the events reconstructed transverse cluster mass $m_{T, c}(\notin, j)$

$$
p_{T, j} / m_{T, c}>2 \text {. }
$$

The resulting $m_{T, c}$ is shown in Fig. 6 together with the projected BR extraction. While the reduction in statistics eventually compensates the enhancement in $S / B$ (compare Fig. 6) b) to Fig. 5(c)), we are able to approximately reconstruct our light Higgs partner $m_{A}=20 \mathrm{GeV}$ from the jet substructure. This is only feasible by exploiting visible final state energy correlations - identical strategies proof unsuitable for scenarios (a), (b) and (d).

A successful and intangible extraction of the light scalar mass from the associated production channel hence depends on a good understanding of the background at small $m_{T, c}$ and 
a sizable branching ratio $H \rightarrow A A \rightarrow$ scenario (c). Current fits limit the invisible branching ratio to be $\mathrm{BR} \lesssim 0.5$. Since all of our signatures are missed in standard searches, our scenarios are constrained by this loose bound, so that there is the possibility to find light scalars in that particular mass range in the near future.

\section{SUMMARY AND CONCLUSIONS}

After the discovery of the Higgs-like resonance at around $125 \mathrm{GeV}$, a further investigation of the resonance's compatibility with the SM expectation is ongoing. A precise measurement of the resonance's couplings to SM matter depends on the treatment of non-standard partial decay widths, which is typically performed in global fit to the quantitative (non-)observation in different exclusive Higgs final states. Non-standard situations are hereby often treated as a nuisance.

Hence, the necessity to investigate non-SM phenomenological situations is imperative: If an indication of a non-zero branching ratio in a specific channel is accessed in a direct measurement, the evidence for physics beyond the SM will be accompanied by an insight in its particular realization as opposed to a mere parametrization of $\Gamma_{\text {inv }}$ in Eq. (1) as part of a global fit. This inevitably comes at the price of a decreased sensitivity (i.e. less stringent limits) which results from investigating a single channel as opposed to combinations and correlations of many.

We have investigated the possibility to constrain nonstandard branching ratios via direct measurements for a broad range of effective models, which contain both invisible and visible final state particles in this paper. Gaining sensitivity to these phenomenological situations is characterized by a number of shortcomings, that range from trigger issues all the way to the Higgs mass reconstruction at a reasonable signalover-background ratio. Since trigger thresholds are typically bound to hardware specifications, we devise an analysis strategy which builds upon associated production and a boosted Higgs final state. As a consequence, we need to deal with an event topology where missing energy is collimated with the visible Higgs decay products in decays $H \rightarrow A A$ with $2 m_{A} \ll 125 \mathrm{GeV}$ (a choice $2 m_{A} \sim 125 \mathrm{GeV}$ exhibits too much $\mathbb{E}_{T}$-jet decorrelation to facilitate signal reconstruction on top of a large background rejection). Recent investigations targeting the reconstruction of $\mathbb{E}_{T}$ based on particle-flow as well as the extraction of fake $\mathbb{E}_{T}$ from a combination of theory and experiment suggest that systematics can be brought under sufficient control to perform such analyses in this particular channel.

We find that, depending on the specific realization of the partially visible Higgs decay (which is characterized by different $\mathbb{E}_{T}$-jet substructure correlations) bounds as good as $\mathrm{BR} \lesssim 10 \%$ can be obtained from a measurement of boosted Higgs final states, applying a rather generic cut set up which serves to obtain a reasonable $S / B$. Constraints can subsequently be obtained from an optimal choice of observable which, depending on the amount of visible final state energy, also allows to partially reconstruct parts of the Higgs' potential decay chain.

Acknowledgements - CE acknowledges funding by the Durham International Junior Research Fellowship scheme. This work was supported in part by the STFC.
[1] The Atlas Collaboration, arXiv:1207.0319, Phys. Rev. Lett. 108, 111803 (2012); Phys. Lett. B 710, 383 (2012); arXiv:1206.0756, arXiv:1206.5971, arXiv:1207.0210, ATLAS-CONF-2012-093; ATLAS-CONF-2012-091; ATLASCONF-2012-092; ATLAS-CONF-2012-098.

[2] The CMS Collaboration, Phys. Lett. B 710 (2012) 26; Phys. Lett. B 710, 91 (2012); Phys. Rev. Lett. 108, 111804 (2012); Phys. Lett. B 710, 403 (2012); Phys. Lett. B 713, 68 (2012); Phys. Lett. B 710, 284 (2012); CMS-PAS-HIG-12-025; CMS-PAS-HIG-12-020; CMS-PAS-HIG-12-015; CMS-PASHIG-12-016; CMS-PAS-HIG-12-017; CMS-PAS-HIG-12-018; CMS-PAS-HIG-12-019.

[3] F. Englert and R. Brout, Phys. Rev. Lett. 13 (1964) 321; P. W. Higgs, Phys. Lett. 12 (1964) 132 and Phys. Rev. Lett. 13 (1964) 508; G. S. Guralnik, C. R. Hagen and T. W. B. Kibble, Phys. Rev. Lett. 13 (1964) 585.

[4] A. Azatov, R. Contino and J. Galloway, arXiv:1202.3415, D. Carmi, A. Falkowski, E. Kuflik, T. Volansky and J. Zupan, arXiv:1207.1718, P. P. Giardino, K. Kannike, M. Raidal and A. Strumia, arXiv:1207.1347, J. Ellis and T. You, arXiv:1207.1693; J. R. Espinosa, C. Grojean, M. Muhlleitner and M. Trott, arXiv:1207.1717, T. Plehn and M. Rauch, arXiv:1207.6108 [hep-ph].

[5] T. Plehn, D. L. Rainwater and D. Zeppenfeld, Phys. Rev.
Lett. 88, 051801 (2002). C. Englert, M. Spannowsky and M. Takeuchi, JHEP 1206, 108 (2012).

[6] B. Coleppa, K. Kumar and H. E. Logan, arXiv:1208.2692 [hep-ph]. S. Bolognesi, Y. Gao, A. V. Gritsan, K. Melnikov, M. Schulze, N. V. Tran and A. Whitbeck, arXiv:1208.4018 [hep-ph]. R. Boughezal, T. J. LeCompte and F. Petriello, arXiv:1208.4311 [hep-ph].

[7] S. Dawson and E. Furlan, arXiv:1205.4733; H. K. Dreiner, J. S. Kim and O. Lebedev, Phys. Lett. B 715 (2012) 199 I. Low, J. Lykken and G. Shaughnessy, arXiv:1207.1093, T. Corbett, O. J. P. Eboli, J. Gonzalez-Fraile and M. C. Gonzalez-Garcia, arXiv:1207.1344, M. Montull and F. Riva, arXiv:1207.1716 [hep-ph]. S. Banerjee, S. Mukhopadhyay and B. Mukhopadhyaya, arXiv:1207.3588, D. Bertolini and M. McCullough, arXiv:1207.4209 [hep-ph]. F. Bonnet, T. Ota, M. Rauch and W. Winter, arXiv:1207.4599, B. Batell, D. McKeen and M. Pospelov, arXiv:1207.6252 [hep-ph].

[8] S. Bock, R. Lafaye, T. Plehn, M. Rauch, D. Zerwas and P. M. Zerwas, Phys. Lett. B 694, 44 (2010); C. Englert, T. Plehn, M. Rauch, D. Zerwas and P. M. Zerwas, Phys. Lett. B 707, 512 (2012); J. R. Espinosa, M. Muhlleitner, C. Grojean and M. Trott, arXiv:1205.6790

[9] C. Englert, J. Jaeckel, E. Re and M. Spannowsky, Phys. Rev. D 85, 035008 (2012). 
[10] S. Chang, R. Dermisek, J. F. Gunion and N. Weiner, Ann. Rev. Nucl. Part. Sci. 58, 75 (2008).

[11] O. J. P. Eboli and D. Zeppenfeld, Phys. Lett. B 495, 147 (2000).

[12] Y. Bai, P. Draper and J. Shelton, JHEP 1207 (2012) 192;

[13] A. Djouadi, A. Falkowski, Y. Mambrini and J. Quevillon, arXiv:1205.3169 [hep-ph].

[14] R. E. Shrock and M. Suzuki, Phys. Lett. B 110, 250 (1982).

[15] U. Ellwanger, J. F. Gunion and C. Hugonie, JHEP 0507, 041 (2005); J. R. Forshaw, J. F. Gunion, L. Hodgkinson, A. Papaefstathiou and A. D. Pilkington, JHEP 0804, 090 (2008).

[16] B. Patt and F. Wilczek, hep-ph/0605188

[17] M. Lisanti and J. G. Wacker, Phys. Rev. D 79 (2009) 115006 [arXiv:0903.1377 [hep-ph]].

[18] J. M. Butterworth, A. R. Davison, M. Rubin, G. P. Salam, Phys. Rev. Lett. 100, 242001 (2008).

[19] A. Falkowski, D. Krohn, L. -T. Wang, J. Shelton and A. Thalapillil, Phys. Rev. D 84, 074022 (2011); C. R. Chen, M. M. Nojiri and W. Sreethawong, JHEP 1011 (2010) 012.

[20] C. Englert, T. S. Roy and M. Spannowsky, Phys. Rev. D 84, 075026 (2011).

[21] I. Lewis and J. Schmitthenner, JHEP 1206 (2012) 072 [arXiv:1203.5174[hep-ph]].

[22] M. Baumgart and A. Katz, arXiv:1204.6032 [hep-ph].

[23] A. Abdesselam, E. B. Kuutmann, U. Bitenc, G. Brooijmans, J. Butterworth, P. Bruckman de Renstrom, D. Buarque Franzosi and R. Buckingham et al., Eur. Phys. J. C 71 (2011) 1661; A. Altheimer, S. Arora, L. Asquith, G. Brooijmans, J. Butterworth, M. Campanelli, B. Chapleau and A. E. Cholakian et al., J. Phys. G G 39, 063001 (2012).

[24] The Atlas Collaboration, Phys. Rev. Lett. 106, 131802 (2011). The CMS Collaboration, Phys. Lett. B 698 (2011) 196.

[25] J. F. Kamenik and C. Smith, Phys. Rev. D 85 (2012) 093017.

[26] R. M. Godbole, M. Guchait, K. Mazumdar, S. Moretti and D. P. Roy, production with gauge bosons,' Phys. Lett. B 571, 184 (2003).

[27] The Atlas collaboration, ATLAS-CONF-2010-057. The CMS collaboration, CMS-JME-10-009.

[28] Z. Bern, G. Diana, L. J. Dixon, F. Febres Cordero, S. Hoche, H. Ita, D. A. Kosower and D. Maitre et al., Phys. Rev. D 84, 114002 (2011).

[29] D. Alves et al. [LHC New Physics Working Group Collaboration], arXiv:1105.2838 [hep-ph].

[30] R. Dermisek, J. F. Gunion and B. McElrath, Phys. Rev. D 76, 051105 (2007).
[31] The Atlas collaboration, ATL-PHYS-PUB-2009-088.

[32] B. Batell, J. Pradler and M. Spannowsky, JHEP 1108, 038 (2011).

[33] N. D. Christensen and C. Duhr, Comput. Phys. Commun. 180, 1614 (2009).

[34] N. D. Christensen, P. de Aquino, C. Degrande, C. Duhr, B. Fuks, M. Herquet, F. Maltoni and S. Schumann, Eur. Phys. J. C 71, 1541 (2011).

[35] T. Gleisberg, S. .Hoeche, F. Krauss, M. Schonherr, S. Schumann, F. Siegert and J. Winter, JHEP 0902, 007 (2009).

[36] S. Dittmaier, S. Kallweit and P. Uwer, Phys. Rev. Lett. 100, 062003 (2008). F. Campanario, C. Englert, S. Kallweit, M. Spannowsky and D. Zeppenfeld, JHEP 1007, 076 (2010). T. Binoth, T. Gleisberg, S. Karg, N. Kauer and G. Sanguinetti, Phys. Lett. B 683 (2010) 154.

[37] P. Nason, S. Dawson and R. K. Ellis, Nucl. Phys. B 303, 607 (1988). W. Beenakker, H. Kuijf, W. L. van Neerven and J. Smith, Phys. Rev. D 40, 54 (1989). S. Moch, P. Uwer and A. Vogt, Phys. Lett. B 714 (2012) 48.

[38] S. Dittmaier et al. [LHC Higgs Cross Section Working Group Collaboration], arXiv:1101.0593 [hep-ph].

[39] The CMS collaboration, CMS PAS PFT-09/001.

[40] M. Cacciari, G. P. Salam and G. Soyez, Eur. Phys. J. C 72, 1896 (2012).

[41] D. Krohn, J. Thaler and L. -T. Wang, JHEP 1002, 084 (2010).

[42] see e.g. The Atlas collaboration ATLAS-CONF-2012-043.

[43] G. Aad et al. [ATLAS Collaboration], JINST 3 (2008) S08003; G. L. Bayatian et al. [CMS Collaboration], J. Phys. G 34 (2007) 995.

[44] T. Plehn, D. L. Rainwater and D. Zeppenfeld, Phys. Rev. D 61, 093005 (2000).

[45] V. D. Barger, T. Han and J. Ohnemus, Phys. Rev. D 37 (1988) 1174.

[46] J. Bagger, V. D. Barger, K.-M. Cheung, J. F. Gunion, T. Han, G. A. Ladinsky, R. Rosenfeld and C.-P. Yuan, Phys. Rev. D 52, 3878 (1995).

[47] T. Junk, Nucl. Instrum. Meth. A 434 (1999) 435. T. Junk, CDF Note 8128 [cdf/doc/statistics/public/8128]. T. Junk, CDF Note 7904 [cdf/doc/statistics/public/7904]. H. Hu and J. Nielsen, in 1st Workshop on Confidence Limits, CERN 2000-005 (2000).

[48] A. L. Read, CERN-OPEN-2000-205. A. L. Read, J. Phys. G G28 (2002) 2693-2704. G. Cowan, K. Cranmer, E. Gross and O. Vitells, Eur. Phys. J. C 71, 1554 (2011). 